\title{
Anaphase initiation in Saccharomyces cerevisiae is controlled by the APC-dependent degradation of the anaphase inhibitor Pds1p
}

\author{
Orna Cohen-Fix, ${ }^{1,4}$ Jan-Michael Peters, ${ }^{2,3}$ Marc W. Kirschner, ${ }^{2}$ and Doug Koshland ${ }^{1,4}$ \\ ${ }^{1}$ Department of Embryology, Carnegie Institution of Washington, Baltimore, Maryland 21210 USA; ${ }^{2}$ Department of Cell \\ Biology, Harvard Medical School, Boston, Massachusetts 02115 USA
}

\begin{abstract}
Anaphase initiation has been postulated to be controlled through the ubiquitin-dependent proteolysis of an unknown inhibitor. This process involves the anaphase promoting complex (APC), a specific ubiquitin ligase that has been shown to be involved in mitotic cyclin degradation. Previous studies demonstrated that in Saccharomyces cerevisiae, Pds1 protein is an anaphase inhibitor and suggested that it may be an APC target. Here we show that in yeast cells and in mitotic Xenopus extracts Pds1p is degraded in an APC-dependent manner. In addition, Pds1p is directly ubiquitinated by the Xenopus APC. In budding yeast Pds1p is degraded at the time of anaphase initiation and nondegradable derivatives of Pds1p inhibit the onset of anaphase. We conclude that Pds1p is an anaphase inhibitor whose APC-dependent degradation is required for the initiation of anaphase.
\end{abstract}

[Key Words: Pds1; anaphase promoting complex; Saccharomyces cerevisiae; Xenopus; ubiquitin-dependent proteolysis]

Received October 2, 1996; revised version accepted October 29, 1996.

The metaphase to anaphase transition is an important step in the eukaryotic cell cycle. This process is believed to be regulated, at least in part, through sister chromatid pairing (for review, see Holm 1994; Miyazaki and OrrWeaver 1994). Sister chromatid pairing is thought to be mediated by cohesion factors that act as a "molecular glue" in associating the sister chromatids along their entire length. These cohesion factors counteract the poleward forces exerted by spindle motors, thereby preventing precocious sister chromatid separation. At metaphase, the replicated and paired sister chromatids congress to the spindle midzone through their bipolar attachments to the spindle microtubules. At anaphase, the cohesion factors are inactivated and the sister chromatids separate synchronously and move to opposite poles by means of their spindle attachment. Misregulation of the cohesion factors could lead to precocious sister chromatid separation. If this occurs prior to the formation of a stable bipolar spindle attachment, the separated chromatids would not be identified as sisters, thereby diminishing the cell's ability to segregate the two sister chromatids to opposite poles. Anaphase initiation is also controlled by checkpoints that arrest the

${ }^{3}$ Present address: Research Institute for Molecular Pathology, Vienna, Austria.

${ }^{4}$ Corresponding authors. cell cycle prior to anaphase in response to spindle or DNA damage, thus allowing time for repair (for review, see Hartwell and Kastan 1994). Following DNA or spindle damage, a premature initiation of anaphase caused by precocious sister chromatid separation would abolish this repair period, leading to chromosome missegragation and the inheritance of damaged DNA.

How the initiation of anaphase is regulated is not clear, but accumulating evidence points to the involvement of proteolysis via the ubiquitin pathway (Murray et al. 1989; Holloway et al. 1993; Irniger et al. 1995). In this process, a ubiquitin-conjugating activity (UBC or E2) together with a ubiquitin ligase activity (also known as E3) attach multiple ubiquitins onto a specific protein substrate, thereby targeting it for degradation (Ciechanover 1994; Hershko et al. 1994; Peters 1994; Murray 1995). The ubiquitin ligase that is thought to be involved in the metaphase to anaphase transition is a multisubunit complex called the anaphase promoting complex (APC) (King et al. 1995) or cyclosome (Sudakin et al. 1995). APC contains Cdc16p, Cdc23p, and Cdc27p (Lamb et al. 1994; Imiger et al. 1995; King et al. 1995), homologs of which have been found in several organisms, including Saccharomyces cerevisiae, Schizosaccharomyces pombe, Xenopus laevis, Drosophila melanogaster, Aspergillus nidulans and human cells (Icho and Wickner 1987; Hirano et al. 1990; Sikorski et al. 1990; O’Donnell et al. 1991; Tu- 
gendreich et al. 1993). Inactivation of these three proteins in vivo leads to a preanaphase arrest /Culotti and Hartwell 1971; Hirano et al. 1988; Tugendreich et al. 1995). These observations suggest that APC-dependent proteolysis inactivates one or more proteins that serve as anaphase inhibitors.

Initially, the only proteins whose degradation was known to be required for passage through mitosis were the mitotic cyclins (Murray et al. 1989). Indeed, mitotic cyclins are largely degraded at the metaphase to anaphase transition, making them good candidates for being the anaphase inhibitor (Murray et al. 1989). The degradation of the mitotic cyclins depends on the presence of a degenerate nine-amino-acid motif, known as the destruction box (Glotzer et al. 1991). The destruction box is required for efficient ubiquitination by APC, and consequently in its absence, cyclins become resistent to APC-dependent degradation (Glotzer et al. 1991; Amon et al. 1994; King et al. 1995; Yu et al. 1996; Zachariae and Nasmyth 1996). Surprisingly, nondegradable cyclin derivatives inhibit the exit from mitosis, rather than anaphase onset (Holloway et al. 1993; Stueland et al. 1993; Surana et al. 1993; van der Velden and Lohka 1993). These findings suggest that the anaphase inhibitor is an APC substrate other than the mitotic cyclins. Indeed, precedence for a noncyclin substrate of APC, Cut2p, has recently been demonstrated in fission yeast (Funabiki et al. 1996b).

If anaphase is regulated by a single inhibitor, one would expect that in its absence, APC function would no longer be required for anaphase initiation. Recently, the product of the $S$. cerevisiae PDS1 gene (for precocious dissociation of sister chromatids) was shown to fit this criterion: A significant fraction of $p d s 1 c d c 16$ and $p d s 1$ $c d c 23$ double mutant cells at the nonpermissive temperature arrested not prior to anaphase, as do the single $c d c$ mutants, but rather at the end of mitosis (Yamamoto et al. 1996b). This suggests that Pdslp is an anaphase inhibitor whose degradation via $\mathrm{APC}$ is required for anaphase initiation. Hence, in the absence of Pdslp, the requirement for APC in anaphase onset is alleviated. Consistent with this is the observation that Pdslp is rapidly degraded at $G_{1}$ (Yamamoto et al. 1996a), a time when APC was shown to be involved in cyclin degradation (Amon et al. 1994). The failure to exit mitosis in the $p d s 1 c d c 16$ and $p d s 1$ cdc23 double mutants probably results from the inability to degrade the mitotic cyclins due to APC inactivation.

The putative anaphase inhibitor may also be involved in the preanaphase arrest conferred by the spindle and DNA damage checkpoints. If this were the case, cells lacking the inhibitor would become hypersensitive to spindle or DNA damaging agents, presumably as a result of the failure to implement a checkpoint arrest. Indeed, cells lacking PDS1 were found to be hypersensitive to nocodazole, a microtubule depolymerizing drug that causes spindle disassembly, and to gamma irradiation, which causes primarily double-stranded breaks in the DNA (Yamamoto et al. 1996b). Furthermore, cells lacking Pds lp failed to maintain sister chromatid cohesion in the presence of nocodazole (although DNA replication and rebudding remained inhibited), and failed to arrest altogether following gamma irradiation (Yamamoto et al. 1996b). These observations suggest that Pdslp is required for the preanaphase checkpoint arrest.

These results prompted us to examine the role of Pdslp in the metaphase to anaphase transition. Specifically, we set out to examine whether Pdslp is degraded in an APC-dependent manner, whether it is degraded at the time of anaphase initiation, and whether its degradation is important for its function as an anaphase inhibitor. To this end, we tested the dependence of Pdslp stability on APC function both in vivo and in vitro. Furthermore, Pdslp derivatives that were resistant to degradation were generated and their effect on cell cycle progression was examined. Based on the results presented here we suggest that Pds1p is indeed an anaphase inhibitor whose APC-dependent degradation is required for the metaphase to anaphase transition.

\section{Results \\ Pds1p is degraded in vivo in an APC-dependent manner}

S. cerevisiae strains defective for APC function arrest at the nonpermissive conditions prior to anaphase, presumably because of the inability to degrade the anaphase inhibitor. The ability of the PDS1 deletion to bypass this arrest (Yamamoto et al. 1996b) suggested that perhaps Pdslp is an APC substrate. To examine whether APC is involved in Pdslp degradation, the stability of Pdslp was examined in $G_{1}$-arrested cells, a phase of the cell cycle in which APC is active in Clb2p degradation (Amon et al. 1994). If Pds lp degradation requires APC function, one would expect that the stability of Pds1p would be greater in $G_{1}$-arrested cells defective for APC function, that is, $c d c 16$ or $c d c 23$ cells, than in $\mathrm{G}_{1}$-arrested wild-type cells. For this purpose PDS1 was placed under control of the GAL1 promoter and introduced on a centromere-based plasmid into wild-type or cdc16-123 cells. These cells were arrested in $\mathrm{G}_{1}$ with $\alpha$ factor at $23^{\circ} \mathrm{C}$ and then shifted to $37^{\circ} \mathrm{C}$ to inactivate the $c d c 16-123$ gene product. Following the temperature shift, PDS1 mRNA was expressed for a period of $30 \mathrm{~min}$ by the induction and subsequent repression of the expression from the GAL1 promoter. The stability of Pdslp was monitored by examining the protein levels at 30 and $60 \mathrm{~min}$ after the repression of the GAL1 promoter.

The results in Figure 1A show that Pdslp is unstable in $G_{1}$-arrested wild-type cells, but is stable in $G_{1}$-arrested cdc16-123 cells. Both strains maintained a $1 \mathrm{~N}$ DNA content as assayed by flow cytometry analysis (data not shown), excluding the possibility that Pdslp was stabilized as a result of an escape from the $G_{1}$ arrest. Furthermore, the PDS1 mRNA was equally unstable in wild-type and mutant cells (Fig. 1B), suggesting that the presence of Pdslp in cdc16-123 cells at 30 and $60 \mathrm{~min}$ following transcriptional repression truly reflects an increase in protein stability and not an alteration in gene 
A.

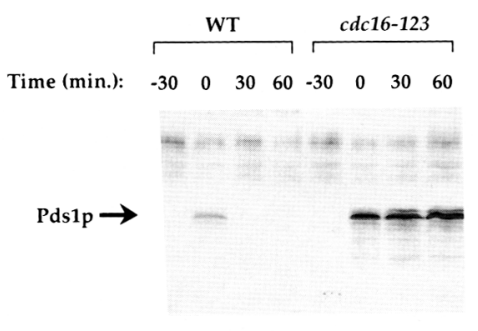

B

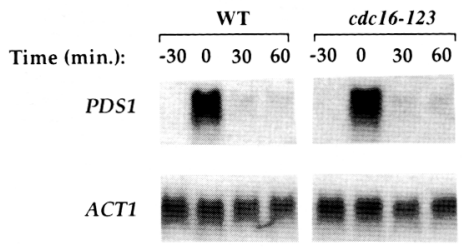

C

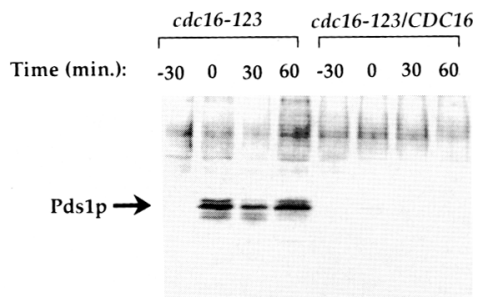

Figure 1. The degradation of Pdslp is dependent on CDC16p function. Wild-type, cdc16-123, and cdc16-123 cells carrying CDC16 on a plasmid (strains K1534, K4438, and K4438/pTK21, respectively/ were transformed by a centomere-based plasmid carrying GAL1-PDS1 (pOC30 for strains in $A$ and B, POC29 for strains in panel $C$ ). All strains were grown in YEP + raffinose to early log phase at $23^{\circ} \mathrm{C}$ and were arrested in $\mathrm{G}_{1}$ with $\alpha$ factor (final concentration $2 \times 10^{-7} \mathrm{M}$ ). When $>90 \%$ of the cells appeared morphologically to be arrested, the cultures were shifted to $37^{\circ} \mathrm{C}$. After $30 \mathrm{~min}$, the expression of PDS1 from the GAL1 promoter was induced for a period of $30 \mathrm{~min}$ by the addition of galactose $(2 \%$ final concentration, at time -30$)$ followed by the subsequent addition of glucose $12 \%$ final concentration, at time 0). Samples of equal cell densities were taken at the indicated time points (while maintaining the $G_{1}$ arrest at the elevated temperature), and assayed by immunoblotting for $\operatorname{Pds} 1 \mathrm{p}(A$ and $C)$ or by Northern analysis for PDS1 and ACT1 mRNA $(B)$. Pdslp specific bands almost always appear as a doublet, with a minor form migrating slightly slower than the major form. The nature of these different forms is currently unknown.

expression or mRNA stability. The enhanced stability of Pdslp in the cdc16 strain was directly related to the deficiency in Cdc16p activity because Pdslp was unstable in $\mathrm{G}_{1}$-arrested cdc16-123 cells that carried wild-type CDC16 on a centromere-based plasmid (Figure 1C). Similarly, Pds1p was stable in $\mathrm{G}_{1}$-arrested $c d c 23-1$ cells but not in the isogenic wild-type strain (data not shown). These results indicate that Pdslp is degraded in a Cdc16p and Cdc23p-dependent manner, suggesting that APC is involved in Pdslp degradation.

Pdslp has a nine-amino-acid motif located near its amino-terminus that resembles the destruction-box motif of B-type cyclins (Yamamoto et al. 1996a; Figure 2A). To examine whether this sequence is a functional destruction box, two types of mutations were generated: $p d s 1-m d b$, in which the conserved RXXL was replaced by AXXA, and $p d s 1-\Delta d b$, in which the sequence coding for the putative destruction box was deleted and replaced by a XhoI restriction site, which encodes a leucine and a glutamic acid (Fig. 2A). To examine the stability of these Pds1p derivatives, the pds1 mutant alleles and the wildtype PDS1 gene were placed under control of a $G A L 1$ promoter on centromere-based plasmids and introduced individually into wild-type cells. Using a strategy similar

A

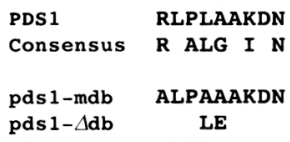

B

Time (min): $\begin{array}{llllllll}0 & 10 & 20 & 30 & 40 & 50 & 60 & 70\end{array}$

PDS1
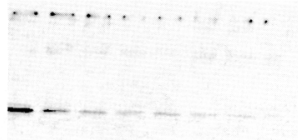

$p d s 1-m d b=-=-\infty=$

$p d s 1-\Delta d b-\infty-\infty=$

Figure 2. Pdslp contains a destruction box motif that is involved in Pdslp degradation by APC. $(A)$ The amino acid sequence (single letter amino acid code) of the Pdslp destruction box starting at position 85 of the protein (full-length Pdslp consists of 373 amino acids) (Yamamoto et al. 1996a). The consensus sequence of the destruction box was derived from Glotzer et al. (1991). Two destruction-box mutant derivatives of PDS1 were generated as described in the text. (B) Wild-type cells

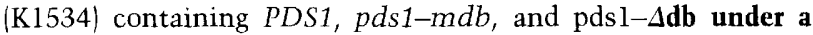
GAL1 promoter on centromere-based plasmids (pOC56, pOC57, and pOC58, respectively) were grown at $30^{\circ} \mathrm{C}$ to early log-phase in YEP + raffinose and arrested in $\mathrm{G}_{1}$ with $\alpha$ factor. When $>90 \%$ of the cells appeared morphologically to be arrested, the expression from the GAL1 promoter was induced for $30 \mathrm{~min}$ as described for Fig. 1. Protein samples were taken every $10 \mathrm{~min}$ and the levels of the Pdslp derivatives were examined by Western blot analysis. 
to the one described above, these cells were arrested in $\mathrm{G}_{1}$ with $\alpha$ factor and the expression of either PDS1 or its mutant derivatives from the GAL1 promoter was induced for $30 \mathrm{~min}$. Samples were taken every $10 \mathrm{~min}$ following the transcriptional shut-off and Pdslp levels were monitored by Western blot analysis. As can be seen in Figure 2B, wild-type Pdslp levels dropped significantly after $20 \mathrm{~min}$, whereas no significant change in the levels of the destruction box mutant gene products was observed throughout the course of the experiment $1>70$ $\mathrm{min})$. These results demonstrate that Pdslp degradation requires an intact destruction box, suggesting that Pdslp is a direct substrate of APC.

\section{Pds1p ubiquitination by APC}

To test whether Pds1p is an APC substrate, the ability of purified APC to promote Pdslp ubiquitination in vitro was examined. Because APC has so far only been purified from Xenopus, we initially tested whether Pdslp is degraded in the crude Xenopus egg extracts, an in vitro system that faithfully reproduces the cell cycle regulation of mitotic cyclin degradation (Murray and Kirschner 1989; Murray et al. 1989; Glotzer et al. 1991; Holloway et al. 1993). Figure 3A shows that ${ }^{35} \mathrm{~S}$-labeled Pdslp was stable in an interphase extract but was rapidly degraded in a mitotic extract, resembling the behavior of cyclin B in these extracts (Glotzer et al. 1991). The mitotic instability of Pdslp was APC-dependent because Pdslp was not degraded in mitotic extracts immuno-depleted of APC with anti-Cdc27p antibodies, whereas depletion with nonspecific control antibodies had no significant effect (Fig. 3B, cf. the level of Pdslp at 60 mins and 10 $\mathrm{min}$ ). Moreover, high molecular mass forms of Pds1p appear following incubation with the untreated and control-depleted extracts, but not following incubation with the APC-depleted extract (Fig. 3B). As will be described below, these forms are likely to be ubiquitinated forms of Pdslp, and their absence in the APC-depleted reaction further suggests that Pdslp is an APC substrate. Mitotic degradation of Pds1p in Xenopus extracts was also dependent on an intact destruction box, as the Pdslp destruction box mutant derivatives were significantly more stable than the wild-type protein (Fig. $3 \mathrm{C}$ ). We noted that the efficiency of Pdslp degradation varied from one extract preparation to another (Figs. 3A-C). Nonetheless, the average half-life of Pdslp was $>90 \mathrm{~min}$ in interphase extracts, $\sim 12 \mathrm{~min}$ in mitotic extracts, and $>60 \mathrm{~min}$ in APC-depleted mitotic extracts. The half lives of both destruction-box PdsIp mutants in mitotic extracts was $>60 \mathrm{~min}$. These results are consistent with the involvement of APC in Pdslp degradation.

To test whether the degradation of Pdslp was a result of ubiquitination by APC, ${ }^{35} \mathrm{~S}$-labeled Pds1p was incubated in reaction mixtures containing immuno-purified Xenopus APC and either one of two E2's, UBC4 or UBCX. The ubiquitin ligase activity of APC can be detected by the appearance of a characteristic ladder of increasingly higher molecular mass products that represent conjugates of the radiolabeled substrate protein
A

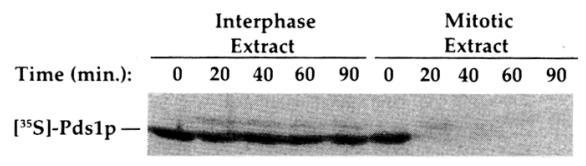

B

Treatment of

Mitotic Extract: Untreated Control-depl. APC-depl.

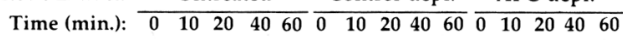

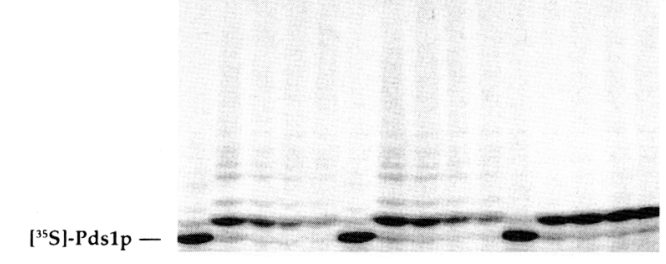

C

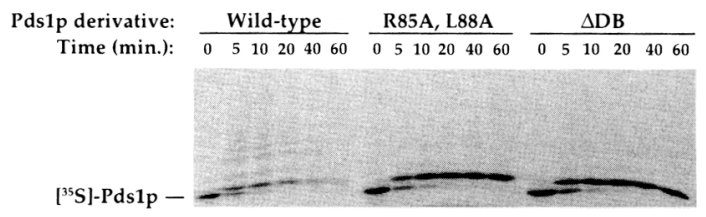

Figure 3. Pdslp is degraded in mitotic Xenopus egg extracts in an APC-dependent manner. $(A)^{35}$ S-labeled Pdslp was incubated in reaction mixtures containing either interphase or mitotic Xenopus egg extracts, as described under Materials and Methods. Samples were removed at the indicated time points and analyzed by SDS-PAGE and PhosphorImaging. $(B)^{35}$ S-labeled Pdslp was incubated in reaction mixtures that contained mitotic Xenopus egg extracts that were either left untreated, depleted with preimmune antibodies (control-depleted) or immunodepleted with anti-Cdc27p antibodies (APC-depleted). Samples were taken at the indicated time points and processed as described in $A$. Occasionally in these extracts Pdslp was initially converted to a slower migrating form, the nature of which is currently unknown (see also panel $C$ ). $(C)^{35}$ s-labeled wildtype Pdslp (from plasmid pOC59) and its destruction-box mutant derivatives $\mathrm{R} 85 \mathrm{~A}, \mathrm{~L} 88 \mathrm{~A}$ (the protein product of $p d s 1-m d b$ from plasmid pOC60) and $\triangle \mathrm{DB}$ (the protein product of $p d s 1-$ $\Delta d b$ from plasmid pOC61/ were incubated in reaction mixtures containing mitotic Xenopus egg extracts and analyzed as described in $A$.

with different numbers of ubiquitin residues (King et al. 1995). As can be seen in Figure 4A, the reconstituted system promoted the formation of high molecular mass conjugates of Pdslp in an APC-dependent reaction that resembled the ubiquitination of an amino-terminal fragment of cyclin B. The molecular masses of modified Pdslp in the ladder (Fig. 4A) differed by an average of $9-\mathrm{kD}$ increments, in agreement with a modification that 

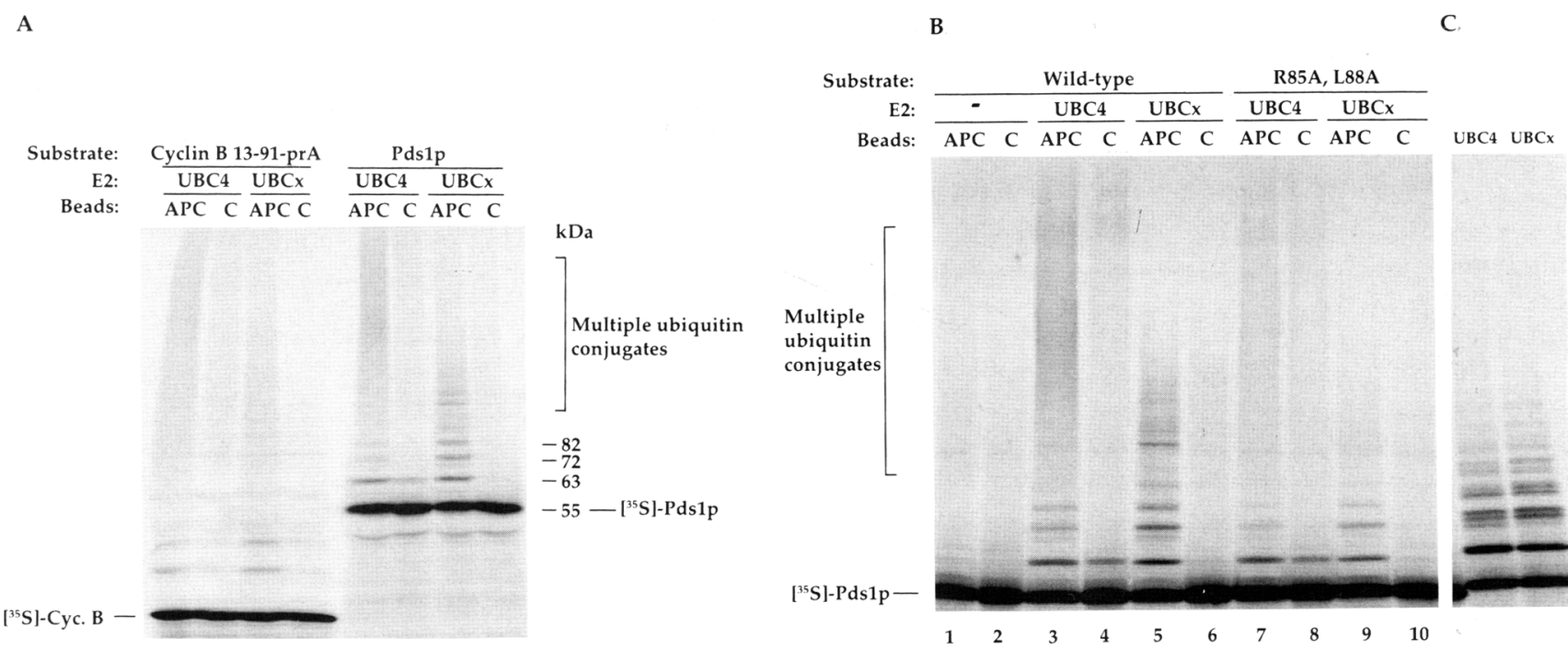

Figure 4. Pdslp is ubiquitinated by APC. $(A)$ Immunopurified APC coupled to Cdc27p antibody beads (APC) and preimmune control beads $(C)$ were assayed for the ability to ubiquitinate ${ }^{35}$ S-labeled Pdslp or a cyclin B-protein A fusion (13-91-prA) in the presence of purified ubiquitin, El and either UBC4 or UBCx. The reaction products were analyzed as described in Materials and Methods. The molecular masses of the modified ${ }^{35}$ S-labeled Pdslp bands that can be individually distinguished are indicated. $(B)^{35} \mathrm{~S}$-labeled Pdslp wild-type (lanes 1-6) and destruction-box mutant (R85A,L88A) (lanes 7-10) were analyzed in APC ubiquitination assays as in $A$. (C) ${ }^{35}$ S-labeled Pdslp was analyzed in APC ubiquitination assays containing methylated ubiquitin. Note that only conjugates of lower molecular mass are formed as compared with the conjugates generated in reactions containing unmodified ubiquitin only (reactions in $B$ and $C$ are from the same experiment).

is a result of the conjugation of one to several ubiquitins. The processivity of the reaction, which is determined by the number of ubiquitins conjugated per substrate molecule, was greater with UBC4 than with UBCx, as can be seen from the distribution of the modified ${ }^{35} \mathrm{~S}$-labeled Pds1p bands (Fig. 4A, note the smear at the top of the $\mathrm{UBC} 4+\mathrm{APC}$ lane). When these reactions were carried out in the presence of methylated ubiquitin, an inhibitor of ubiquitin chain elongation, Pdslp forms of much lower molecular mass were generated (Fig. 4C), confirming that the slow migrating Pdslp forms represent ubiquitinated Pdslp.

As mentioned above, the stability of Pdslp in the mitotic Xenopus egg extracts could be enhanced by mutating its destruction box. To test whether this effect resulted from inefficient ubiquitination by APC, the ubiquitination of ${ }^{35} \mathrm{~S}$-labeled wild-type Pdslp and the Pdslp destruction-box mutant R85A, L88A was examined in reconstituted APC reaction mixtures (Fig. 4B). In reactions containing APC and UBC4, the ubiquitination of the Pdslp destruction box mutant was significantly less than that of wild-type Pdslp /cf. lanes 3 and 7, note the absence of the multiple ubiquitin conjugated forms in lane 7). In these reactions the amount of modified wildtype Pdslp was five-fold greater than the amount of modified Pdslp destruction-box mutant, as determined by densitometry. Similarly, in reactions containing UBCX, the amount of modified wild-type Pdslp was three-fold higher than the amount of modified Pdslp destruction box mutant (cf. lanes 5 and 9). Mutation of the Pds1p destruction box did not result in the complete loss of ubiquitination, as has been observed previously with similar destruction box mutants of cyclin B (Yu et al. 1996), but rather led to a significant reduction in the number of ubiquitins that were conjugated per protein. This suggests that mutations in the destruction box reduce the affinity between APC and its substrates. Proteins with shorter ubiquitin chains are not efficiently recognized by the 26 S proteosome (Chau et al. 1989), resulting in their stabilization. Taken together, these results strongly suggest that Pds1p is a substrate of APC and that efficient recognition of Pdslp by APC is mediated by the Pdslp destruction box.

\section{Pds1p is degraded prior to anaphase initiation}

Because Pdslp appeared to be a substrate of APC, it was of interest to determine when in the cell cycle Pds $1 p$ is degraded, and whether its degradation correlates with the stages of the cell cycle at which APC is active, namely, from early mitosis to the $\mathrm{G}_{1} / \mathrm{S}$ boundary (Amon et al. 1994). As endogenous levels of Pdslp could not be detected with the anti-Pdslp antibodies available, the wild-type PDS1 gene was replaced with a construct encoding a hemagglutinin epitope-tagged version of $P D S 1$, PDS1::HA. The PDS1::HA allele was found to fully complement the known $p d s 1 \Delta$ phenotypes (data not shown). To determine when during the cell cycle Pdslp is present, $P D S 1:: H A$ under the control of wild-type PDS1 promoter was introduced into a $c d c 15$ strain, which arrests late in mitosis when grown at $37^{\circ} \mathrm{C}$. The levels of Pdslp-HA were then examined in these cells when ar- 
rested in $G_{1}$ (by treatment with $\alpha$ factor), $M$ (by treatment with nocodazole), or in late $M$ (by growth at $37^{\circ} \mathrm{C}$ ). Pdslp-HA was present in $M$ phase-arrested cells, but not in $G_{1}$ cells, and the level of Pdslp-HA in late $M$ was greatly reduced (Fig. 5A). The reduced level of Pdslp in the $c d c 15$ cells arrested at $37^{\circ} \mathrm{C}$ was not a result of a general instability of the protein at the elevated temperature because Pdslp-HA was stable in wild-type, $c d c 16$, and $c d c 13$ cells that were incubated for $3 \mathrm{hr}$ at $37^{\circ} \mathrm{C}$ /data not shown).

To determine the pattern of Pdslp expression with greater precision, PDS1::HA cells were released synchronously from a hydroxyurea (HU) arrest, in which cells are blocked in early $\mathrm{S}$ phase as large budded cells with a single nucleus. Cell morphology, as well as Pdslp-HA and $\beta$-tubulin levels were determined at various time points following the release. As can be seen in Figure 5B, the level of Pdslp-HA decreased at $75 \mathrm{~min}$ following the release and rose again at $\sim 30 \mathrm{~min}$ later. When comparing this pattern with the cellular morphology (Fig. 5C), one can observe a strong correlation between the decrease in the level of Pdslp-HA and the decrease in the fraction of cells with a preanaphase morphology (large-budded cells with a single nucleas). This suggests that Pdslp is degraded as cells enter anaphase. The level of Pdslp-HA increases prior to the increase in the fraction of preanaphase cells in the subsequent cell cycle, in agreement with the finding that Pdslp is present in small-budded, late $G_{1}$ cells (Yamamoto et al. 1996a; see below).

Fusing the HA epitope to Pdslp also enabled us to use indirect immunofluorescence to visualize the endogenous levels of Pdslp in unperturbed cycling cells. An asynchronous population of wild-type diploid cells, homozygous for PDS1::HA, was fixed and immunostained for Pds1p-HA. These cells were then examined for bud morphology, DNA distribution, and positive staining for the HA epitope, thus correlating the cell cycle stage with the presence of Pdslp-HA. In cells that were stained for the antigen, a general nuclear staining could be observed (Fig. 6). Presently, we cannot determine whether this signal is distributed throughout the nucleoplasm or is confined to the chromosomes. No signal was observed in the control PDS1 cells, demonstrating that this staining was specific to the HA antigen. Strikingly, the HA-specific staining was restricted to cells that were in between the late $G_{1}$ (small budded cells) and preanaphase (large budded cells with a single nucleus) stages of the cell cycle. HA-specific staining was not observed in anaphase or telophase cells and only rarely detected in unbudded cells. These results suggest that in normal cycling cells, Pdslp is degraded shortly before or at the onset of anaphase, and reappears at the time of bud emergence, as was suggested from our analysis of staged cells (Fig. 5).

\section{Pds1p degradation is required for the initiation of anaphase}

Our results suggest that Pdslp is degraded via APC ubiquitination at the time of anaphase initiation. It was therefore of interest to determine whether this degrada-

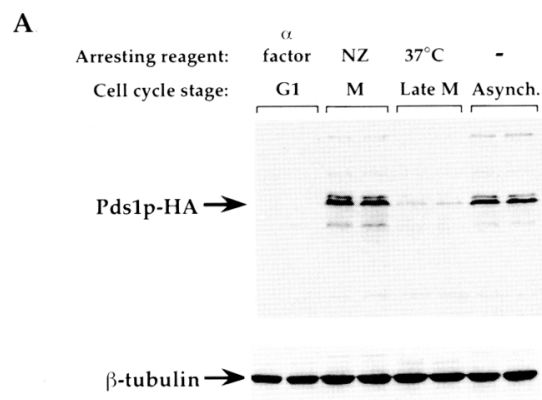

B
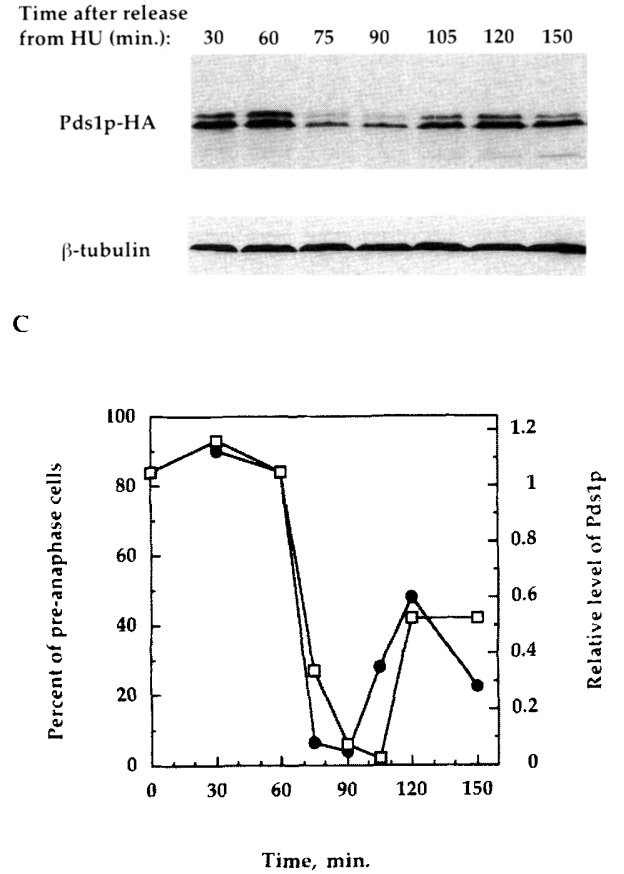

Figure 5. Pdslp is present at $\mathrm{G}_{2} / \mathrm{M}$ but not in late $M$ or $\mathrm{G}_{1}$. $(A)$ A cdc15-2 strain containing PDS1::HA (OCF1515-7D) was grown to early $\log$ phase at $23^{\circ} \mathrm{C}$ and then divided into four separate cultures. One culture was left untreated and the remaining three were arrested in either $G_{1}$ with $\alpha$ factor (final concentration $2 \times 10^{-7} \mathrm{M}$ ), in $\mathrm{M}$ with nocodazole (final concentration $15 \mu \mathrm{g} / \mathrm{ml}$ ) or in late $\mathrm{M}$ (telophase) by temperature shifting to $37^{\circ} \mathrm{C}$. The cultures were grown under these conditions for $4 \mathrm{hr}$ at which time the percentage of cells that appeared arrested at the expected cell cycle stage was $93 \%$ for the $\alpha$ factor treated culture, $91 \%$ for the nocodazole treated culture, and $90 \%$ for the temperature shifted culture. Samples of equal cells densities were harvested and analyzed for Pds1p-HA (upper panel) or $\beta$-tubulin (lower panel) by Western blot analysis. Duplicate samples from identical conditions were fractionated side by side. $(B, C)$ Wild-type cells harboring PDS1::HA (OCF1521.0) were grown at $30^{\circ} \mathrm{C}$ and arrested in early $S$ phase with $\mathrm{HU}$. When $>90 \%$ of the cells appeared morphologically to be arrested, the cells were released from the arrest by washing out the drug. Samples were taken at the indicated time points and analyzed for Pdslp-HA and $\beta$-tubulin by Western blot analysis, and for cell morphology by DAPI staining. In panel $C$, the percentage of preanaphase cells [large-budded with a single nucleus ( $\square$ )] and the relative amount of Pds1p-HA [normalized to the amount of $\beta$-tubulin (O)] is presented for each time point. 


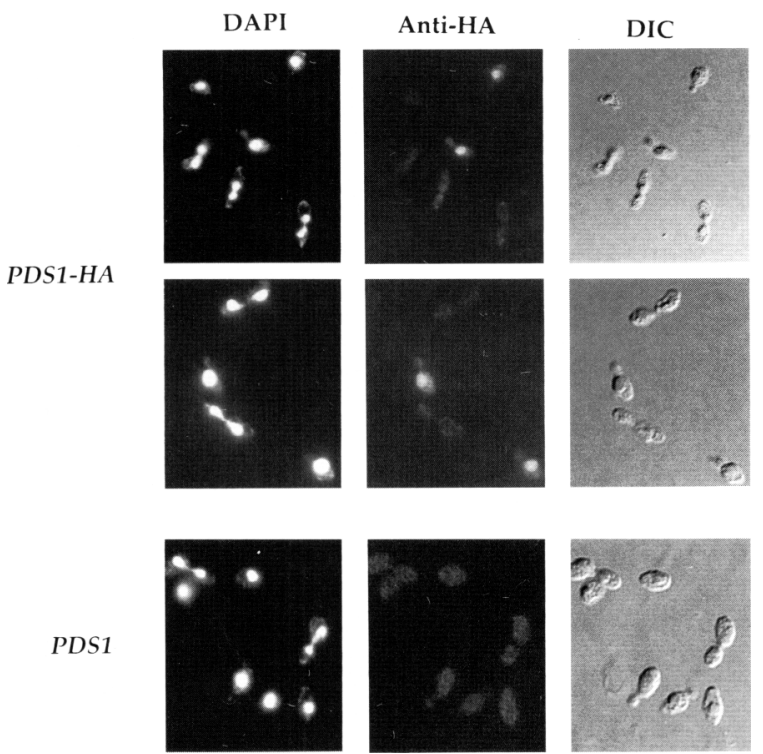

Figure 6. Pdslp is undetectable in anaphase, telophase, and unbudded cells. A PDS1::HA homozygous diploid strain (OCF1507) and a wild-type PDS1 diploid strain (OCF1511) were grown to early $\log$ phase at $30^{\circ} \mathrm{C}$ and processed for immunofluorescence as described in Materials and Methods. It should be noted that the distribution of cell types as seen in the PDS1::HA strain panels does not reflect the cell type distribution in this culture /which was identical to that of the wild type PDS1 culture). Rather, these particular fields were chosen in order to demonstrate the type of signal seen in cells of different stages of the cell cycle.

tion is important for the transition from metaphase to anaphase. If this were the case, one would expect that the expression of nondegradable Pdslp derivatives would be deleterious to the cell, as the cells would not be able to initiate anaphase properly. Indeed, attempts to transform wild-type cells with centromere-based plasmids carrying $p d s 1-m d b$ or $p d s 1-\Delta d b$ expressed from the wild-type PDS1 promoter did not yield viable transformants (data not shown), suggesting that even low levels of the destruction box-mutated Pds1p were lethal. To overcome the toxicity of the Pdslp destruction-box mutants, the expression of the pds1 destruction-box mutants, as well as that of PDS1, was placed under the control of the GAL1 promoter. As expected, under constitutive inducing conditions the expression of the destruction box pds1 mutants, as well as that of the wildtype PDS1, was found to be lethal (data not shown). To examine the effect of the destruction box Pdslp mutants on the cell cycle, wild-type cells and cells carrying one of the galactose-inducible constructs described above were arrested in early $\mathrm{S}$ phase with $\mathrm{HU}$ and the expression from the GAL1 promoter was induced for $1 \mathrm{hr}$. Following the transcriptional repression, the HU was removed and the cells were released from the S-phase block. Cell cycle progression and Pdslp levels were monitored.

The rate of recovery from the HU block was initially the same for the wild-type cells and cells that had transiently overexpressed either PDS1, $p d s 1-m d b$ or $p d s 1-$ $\Delta d b$, as all cultures gave rise to a similar fraction of cells with a $2 \mathrm{~N}$ DNA content within $40 \mathrm{~min}$ (Fig. 7A)

The cellular morphology, as determined by $4^{\prime}, 6^{\prime}-$ diamidino-2-phenylindole (DAPI) staining (Fig. 7B), revealed that the wild-type cells proceeded through the cell cycle, such that $60 \mathrm{~min}$ following the release from the HU arrest $\sim 60 \%$ of the cells were in either anaphase or telophase. However, cells that had transiently overexpressed either form of the Pdslp destruction box mutant failed to initiate anaphase (Fig. 7B), and remained arrested at the preanaphase stage for at least $1 \mathrm{hr}$ after the wild-type strain had initiated anaphase. This was evident by their dumbbell shape, their $2 \mathrm{~N}$ DNA content, their undivided nucleus with a short spindle, and the lack of sister chromatid separation (Fig. 7A,D,E). It is noteworthy that when cells continuously overexpressed the Pdslp destruction box mutants for $>6 \mathrm{hr}$, virtually no anaphase or telophase cells were observed, although with time a small population of cells that appeared to be aploid accumulated, as if following an initial arrest some cells underwent cytokinesis in the absence of nuclear division (data not shown).

Cells that had transiently overexpressed wild-type Pds1p while arrested in early $S$ phase exhibited a delay in initiating anaphase, as seen by the delay relative to the wild-type cells in the decline in the fraction of $G_{2} / M$ figures, in the appearance of anaphase/telophase cells, and in the appearance of cells with $1 \mathrm{~N}$ DNA content (Fig. 7A,B). In these cells, the appearance of a significant fraction of cells with anaphase/telophase morphology ( $\sim 80$ min following the release from the HU block) correlated with a significant decrease in the level of Pdslp (Fig. 7C), further supporting the notion that Pdslp degradation is required for the initiation of anaphase. Taken together, these results demonstrate that nondegradable Pds lp derivatives block anaphase initiation and suggest that the degradation of Pds1p via APC-dependent ubiquitination is required for the initiation of anaphase.

Could the inhibitory affect of the nondegradable Pds1p derivatives be the result of a nonspecific inhibition of APC function? To address this question we wanted to test the stability of an APC substrate in the presence of the Pds1p destruction box mutants. Because we had shown that Pdslp is an APC substrate, we examined whether wild-type Pdslp is degraded at the preanaphase arrest induced by a Pdslp destruction-box mutant. For this purpose, the GAL1-pds1-mdb construct was introduced into cells carrying PDS1::HA. These cells were arrested in early $\mathrm{S}$ phase with $\mathrm{HU}$ and the expression from the GAL1 promoter was induced for $1 \mathrm{hr}$, as described above. Following the repression of expression from the GAL1 promoter, the cells were released from the HU block, and the Pds $1 \mathrm{p}-\mathrm{HA}$ and $\beta$-tubulin levels as well as cell morphology were monitored (Fig. 8B). As controls, PDS1::HA cells lacking the GAL1 inducible construct were also arrested in $\mathrm{HU}$, treated with galactose, and released in the presence of $\alpha$ factor (Fig. 8A) or in the presence of nocodazole (Fig. 8C). As can be seen in 
Cohen-Fix et al.
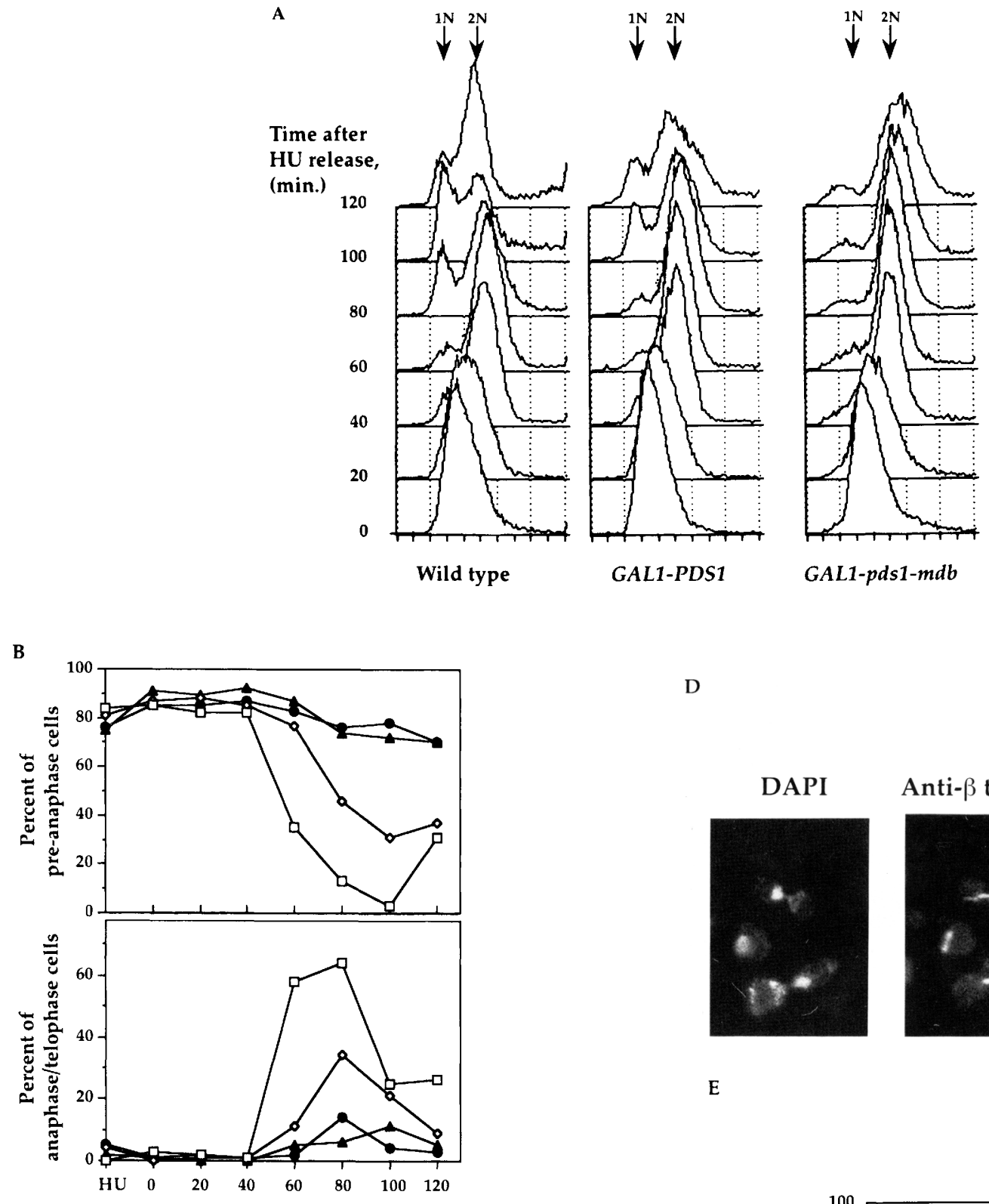

D

Time, $\min$.

C

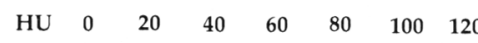

GAL1-PDS1

GAL1-pds1-mdb

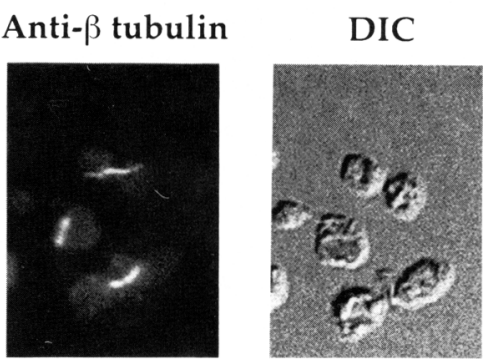

E

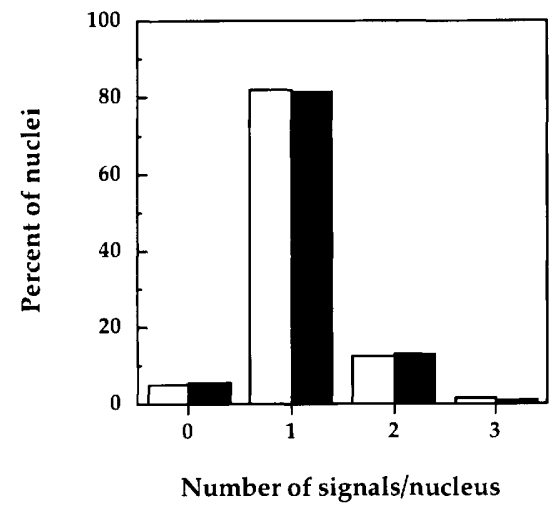

Figure 7. (See facing page for legend.) 
A

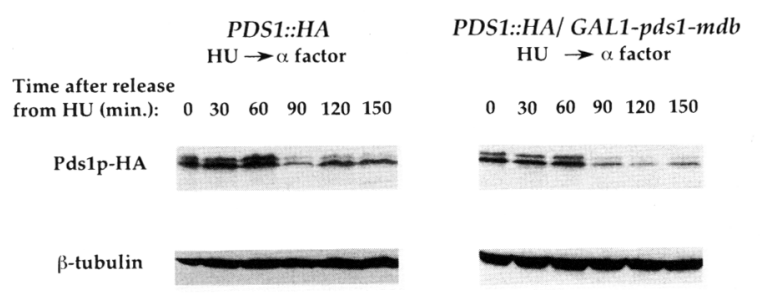

C

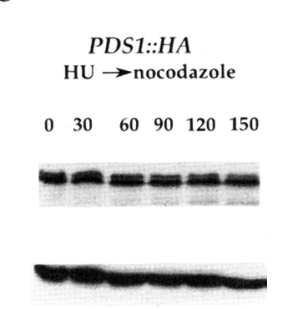

Figure 8. Pdslp destruction-box mutants do not inhibit APC activity. PDS1::HA cells [OCF1521.0) and PDS1::HA cells containing GAL1-pds1-mdb (1521.2) were arrested in early S phase with HU. When $>85 \%$ of the cells appeared morphologically to be arrested, galactose was added to all cultures. After $1 \mathrm{hr}$, the cells were washed with YEP + glucose, to shut off expression from the GAL1 promoter and to release the cells from the HU block, and released from the HU block in the presence of alpha factor $(A, B)$ or in the presence of nocodazole $|C\rangle$. Samples were taken every $30 \mathrm{~min}$ and examined for cell morphology (by DAPI staining) and levels of Pds1p-HA and $\beta$-tubulin (by Western blot analysis). For each culture, the relative amount of Pdslp-HA (normalized to the level of $\beta$-tubulin and assigning the level of Pds1p-HA at time 0 as 1.0 ) is compared with the fraction of preanaphase cells (lower panels). The increase in the

$\rightarrow \square-$ Percent of pre-anaphase cells amount of Pdslp-HA seen at the late time points in $A$ probably resulted from cells that had escaped the $\alpha$ factor arrest, as was evident
by cell morphology (data not shown). by cell morphology (data not shown).

Figure $8 \mathrm{~A}$, the level of Pdslp-HA in otherwise wild-type cells decreased when cells exited the preanaphase state. In the presence of the destruction-box Pds1p mutant, the cells did not initiate anaphase, but nonetheless the levels of Pds lp-HA decreased at a rate comparable to that observed in wild-type cells undergoing anaphase /cf. Fig. $8 \mathrm{~A}, \mathrm{~B})$. The degradation of Pds1p-HA in the presence of the Pds $1 p$ destruction box mutant was not a result of the prolonged preanaphase arrest because the Pdslp-HA levels remain high in cells arrested prior to anaphase by treatment with nocodazole (Fig. 8C). These results indicate that APC is active in the presence of the Pdslp destruction-box mutants. Moreover, this strongly suggests that the inability to initiate anaphase is directly caused by the inability to degrade the Pdslp destructionbox mutants.

\section{Discussion}

Several lines of evidence implicate APC in Pds1p degradation: First, Pdslp, which is normally not detected in extracts of $\mathrm{G}_{1}$-arrested wild-type cells, is stabilized in $\mathrm{G}_{1}$-arrested cells that are impaired in APC function. The APC-dependent degradation of Pdslp in $G_{1}$ is similar to that observed for $\mathrm{Clb} 2 \mathrm{p}$, an established APC substrate (Amon et al. 1994). Second, mutations in the putative destruction-box motif of Pds1p enhanced protein stabil-

Figure 7. The presence of Pdslp coincides with the inability to initiate anaphase. A wild-type strain (OCF1517.0) and strains containing GAL1-PDS1 (OCF1517.1), GAL1-pds1-mdb (OCF1517.2), or GAL1-pds1- $\Delta d b$ (OCF1517.3) were grown to early log phase at $30^{\circ} \mathrm{C}$ in YEP + raffinose and were arrested at early S phase with $\mathrm{HU}$ (final concentration $0.1 \mathrm{M}$ ). When $>85 \%$ of the cells appeared morphologically to be arrested $(\sim 3.5 \mathrm{hr}$ following the addition of $\mathrm{HU})$, the expression from the GAL1 promoter was induced for $1 \mathrm{hr}$ by the addition of galactose $(2 \%$ final concentration). The cells were then harvested, washed twice in YEP + glucose, and then resuspended YEP + glucose in the original volume. Samples where taken every 20 min and processed for FACS analysis, DAPI staining, Western blot analysis, immunofluorescence, and FISH as described in Materials and Methods. (A) FACS analysis of the DNA content in wild-type, GAL1-PDS1, and GAL1-pds1-mdb strains from samples taken at the indicated time points. $(B)$ The cell-type distribution of wild-type $([]), G A L 1-P D S 1(\diamond), G A L 1-p d s 1-m d b(0)$, and $G A L 1-p d s 1 \Delta d b(\mathbf{A})$ at the HU arrest and following the release and GAL1-dependent transcription shut off (time 0). Only the fraction of large-budded cells with a single nucleus (preanaphase cells, top) and large-budded cells with separated DNA masses (anaphase and telophase cells, bottom) are shown. (C) The protein levels of Pdslp (top) or Pdslp(R85A L88A) (the product of $p d s 1-m d b$, bottom) at the indicated time points as detected by Western blot analysis using anti-Pds $1 \mathrm{p}$ antibodies. $(D)$ DAPI staining and indirect immunofluorescence using anti- $\alpha$-tubulin antibodies of $G A L 1-p d s 1-m d b$ cells taken $100 \mathrm{~min}$ after HU release and treated as described in Materials and Methods. (E) FISH analysis of the GAL1-pds1-mdb cells at the time of the HU arrest (open bars) and $2 \mathrm{hr}$ following the GAL1-transcription shut-off and release from the HU block (solid bars). Each FISH signal corresponds to one sister chromatid or two sister chromatids that have not separated (Guacci et al. 1994). Had the sister chromatids separated, an increase in the number of nuclei with two FISH signals would have been expected (Guacci et al. 1994; Yamamoto et al. 1996b). The probe used was of a cetromere proximal probe of chromosome 16. Similar results were obtained when a centromere distal probe of chromosome 16 was used (data not shown). The total number of nuclei counted was 300 (for the $\mathrm{HU}$-arrested cells) and 600 (for the $2 \mathrm{hr}$ time point). 
ity even in APC-proficient strains, consistent with the requirement for a destruction box in the APC-dependent degradation of Clb2p (Surana et al. 1993). Third, Pdslp is degraded in Xenopus egg extracts in an APC-dependent manner. Finally, in a reconstituted system, the Xenopus APC was capable of promoting Pdslp ubiquitination in a manner that was dependent on the presence of a functional destruction box. Based on these results we conclude that Pdslp is a direct target of APC.

Using both immunofluorescence and immunoblotting analysis we were able to establish that Pdslp is present in cells from late $G_{1}$ and until the point of anaphase initiation, and that the time of its degradation correlates with the time of anaphase onset. Moreover, we have shown that not only is Pdslp degraded in an APC-dependent manner, but that its degradation is important for its function as an anaphase inhibitor. Indeed, when Pdslp was rendered nondegradable by inactivating its destruction box, anaphase did not occur. Furthermore, when Pdslp was transiently overexpressed, there was a correlation between the delay in anaphase initiation and the extent of Pdslp degradation. We conclude that the degradation of Pdslp via APC ubiquitination is an integral part of its biological function as an anaphase inhibitor.

An alternative explanation for the inhibitory effect of the nondegradable Pdslp derivative is that anaphase was inhibited not because of the inability to degrade Pdslp per se, but because of competition of the Pdslp destruction-box mutant with other putative APC substrates. Although we cannot eliminate this possibility, it is unlikely. We demonstrated that in the presence of the Pdslp destruction-box mutants APC was active, as seen by the degradation of endogenous Pdslp-HA while the cells were maintained in the preanaphase arrest /this study). This is similar to the case of the cyclin B destruction box mutants, where it has been shown that wildtype cyclin B was degraded in the presence of stable cyclin B derivatives (Glotzer et al. 1991; Luca et al. 1991). The reduced levels of ubiquitination of the destruction box mutants observed in vitro suggest that they have lower affinity for APC and, hence, are likely to be poor competitors for APC function. Another indication that Pdslp itself needs to be degraded at the metaphase to anaphase transition is the ability of a pds1 $\Delta$ to bypass the cdc16 or cdc23 preanaphase arrest (Yamamoto et al. 1996b). Taken together, we suggest that the inability to initiate anaphase in the presence of the Pdslp destruction box mutants was not the result of competition for APC activity, but resulted directly from the inability to degrade this form of Pdslp.

In a previous study of PDS1 (Yamamoto et al. 1996a) GAL1-PDS1 expression was induced in nocodazole-arrested cells from which the drug was removed subsequently. Under these conditions Pdslp was detected in telophase cells, but was absent from $G_{1}$ cells. This result differs from the finding presented here that Pdslp-HA was not detected by immunofluorescence in telophase cells. This difference may be the result of the excess amounts of Pdslp caused by its overproduction. Alternatively, Pdslp produced in nocodazole-arrested cells may be partially exempt from degradation. We suggest that prior to anaphase initiation, $\mathrm{Pdslp}$ is present in a complex, and that this complexed form is specifically targeted for APC-dependent degradation at the time of anaphase onset. In nocodazole-arrested cells, ectopically expressed Pdslp may be excluded from this previously assembled complex and spared from degradation. This exclusion is consistent with a preliminary result that expression of a Pdslp destruction box mutant in nocodazole-arrested cells does not inhibit anaphase initiation upon removal of the drug $/ O$. Cohen-Fix and D. Koshland, unpubl.). It is, therefore, possible that during mitosis, APC recognizes its targets as part of larger complexes, a feature that may also contribute to the specificity of APC-dependent degradation. Upon exiting mitosis, both complexed and noncomplexed Pdslp are degraded, perhaps to set the stage for a new cell cycle.

Is Pds1p the only negative regulator of anaphase? Three observations argue against this possibility. First, assuming that anaphase onset must be regulated in every cell cycle, $p d s 1 \Delta$ strains are still viable, albeit very sick (Yamamoto et al. 1996a). Second, in the pds1s cdc16 or pds $1 \Delta$ cdc23 double mutants only $\sim 50 \%$ of the cells can bypass the preanaphase block of the single $c d c 16$ or cdc23 strains (Yamamoto et al. 1996b). Finally, in the absence of Pdslp only $\sim 50 \%$ of the cells show premature sister chromatid separation when arrested in nocodazole (Yamamoto et al. 1996b). These results suggest that perhaps there is another anaphase inhibitor that is also an APC substrate and that partially complements Pdslp activity in inhibiting anaphase. No homologs of Pdslp were identified in $S$. cerevisiae and so far none have been found in other organisms. However, the Cut2 gene of $S$. pombe has been shown recently to have properties similar to those of Pds1p: Cut2p contains two destruction box motifs, and its degradation requires a functional Cut9p, a homolog of Cdc16p and, therefore, likely a subunit of fission yeast APC (Funabiki et al. 1996b). In addition, Cut $2 p$ derivatives that lack the destruction box inhibit the onset of anaphase (Funabiki et al. 1996). Interestingly, Cut2p associates with Cutlp (Funabiki et al. 1996a), a homolog of the S. cerevisiae gene ESP1 (Uzawa et al. 1990; McGrew et al. 1992) with which Pds1p may have genetic interactions (V. Guacci, O. Cohen-Fix, and D. Koshland, unpubl.). The distribution of the charged amino acids in Cut $2 p$ resembles that of Pds1p, in that there is clustering of acidic and basic residues (Uzawa et al. 1990; Yamamoto et al. 1996a). However, there is no extensive sequence homology between the two proteins. The localization of Cut $2 p$ and Pds $1 p$ is different: Cut $2 p$ is associated with the spindle (Funabiki et al. 1996b), whereas Pdslp shows a general nuclear localization. Moreover, Cut2p is essential whereas Pdslp is not. Some phenotypes of $p d s 1$ mutant strains have yet to be examined in cut 2 mutants. These include the examination of whether cut 2 mutants have a defect in maintaining sister chromatid cohesion when arrested in metaphase, whether the absence of Cut $2 \mathrm{p}$ alleviates the requirement for APC function at the metaphase to anaphase transition, and whether cut 2 mutants are sensitive to ionizing 
radiation. We are currently investigating whether Cut $2 p$ is functionally homologous to Pds1p.

Our results demonstrate that APC can ubiquitinate a protein other than the mitotic cyclins. Genetic data imply the existence of additional substrates, such as Cut $2 p$ (Funabiki et al. 1996b) and Aselp (David Pellman, pers. comm.). These findings suggest that APC is not involved in the degradation of a single regulator that in turn activates a cascade of events. Rather, APC is involved in the degradation of multiple regulators at different stages of mitosis (i.e., anaphase initiation by the degradation of Pdslp and the exit of mitosis by the degradation of Clb2p), as predicted by Holloway et al. (1993). This raises the interesting question of how APC recognizes different substrates, and what provides the temporal regulation where different substrates are degraded at different stages of the cell cycle. In this regard, it will be interesting to examine mitotic cyclin degradation in cells arrested prior to anaphase by the Pdslp destruction-box mutant.

Although these studies provide significant insights into Pdslp regulation, we know little about how Pdslp acts as an anaphase inhibitor. Pdslp may be a structural component of sister chromatid cohesion and the inhibition of sister chromatid separation may in itself be sufficient to inhibit the onset of anaphase. Alternatively, Pdslp may regulate cohesion or other cellular functions that in turn lead to a preanaphase arrest. If this is the case, the requirement for Pdslp degradation in the initiation of anaphase raises the possibility that the cohesion factor itself is not degraded at the onset of anaphase and is inactivated by a different mechanism. Pdslp is also essential for exerting a preanaphase DNA damage checkpoint arrest (Yamamoto et al. 1996b), and it will be of interest to determine whether Pdslp inhibits anaphase in the same manner in the case of the DNA damage checkpoint as it does under normal growth conditions. The identification of cellular components with which Pdslp interacts should help in elucidating the activity of Pdslp at the molecular level.

\section{Materials and methods}

\section{Plasmids and strains}

Plasmid: pTK21 was a generous gift from R. Wickner (National Institutes of Health, Bethesda, MD) (Icho et al. 1987). pOC29 and pOC30 carry the PDS1 open reading frame plus 80 bp of $5^{\prime}$ flanking sequence ligated into the SmaI site of p415GAL1 and p416GALl, respectively (Mumberg et al. 1994). pOC52 is a pUC19-based plasmid carrying PDS1::HA and URA3, which was integrated at an $X b a \mathrm{I}$ site located $3^{\prime}$ of the PDS1 open reading frame. PDS1::HA is an in frame fusion of the triple HA repeat at the $B g I I I$ site of $P D S 1$ (position 520 relative to the first open reading frame base). pOC56, pOC57, and pOC58 carry $P D S 1, p d s 1-m d b$, and $p d s 1-\Delta d b$, respectively ligated into the SmaI site of p416GAL1 (Mumberg et al. 1994). pOC59, pOC60, and pOC61 were constructed by ligating PDS1, pds1-mdb, and $p d s 1-\Delta d b$, respectively, into the SmaI site of pCS2 ${ }^{+}$(Turner and Weintraub 1994). Strains K1534 (MATa ade2 trp1 can1 leu2 his3 ura3 bar) and K4438 (as K1534 but cdc16-123) were a generous gift from $\mathrm{K}$. Nasmyth (Forschung Institut für Molekulare
Pathologie, Vienna, Austria). OCF1511: Mata/Mata bar1/bar1 ura3/ura3 leu2/LEU2 TRP1/trp1. OCF1507: as OCF1511 except also PDS1::HA URA3/PDS1::HA URA3. OCF1517.0, OCF1517.1, OCF1517.2, and OCF1517.3 are as K1534 except that LEU2, PDS1 LEU2, pds1-mdb LEU2, or pds1-Adb LEU2 were integrated at $1 e u 2$ of $\mathrm{K} 1534$, respectively. OCF1515-7D: MATa bar1 cdc15-2 PDS1::HA URA3 (ura3). OCF1521.0: Mata PDS1::HA URA3 (ura3) leu2::LEU2 his3 trp1 ade2. OCF1521.2: as OCF1521.0 except leu2::GAL1-pds1-mdb LEU2. The pds1 destruction-box mutants were constructed using the Transformer Mutagenesis kit by Clontech (Palo Alto, CA) according to manufacturer's instructions.

\section{Media and reagents}

All media for yeast cultures were prepared as described previously (Rose et al. 1990). YEP media contained $1 \%$ yeast extract, $2 \%$ bactopeptone, and $2.5 \mathrm{mg} / 1$ adenine. Carbon sources/glucose, raffinose, or galactose) were used at $2 \%$ final concentration. $\alpha$ factor, $\mathrm{HU}$, and 4 DAPI were purchased from Sigma Chem. Co. (St. Louis, MO). Nocodazole was purchased from Aldrich Chem. Co. (Milwaukee, WI).

In vitro degradation and ubiquitination assay

$\left[{ }^{35} \mathrm{~S}\right]$ methionine labeled proteins were generated from pOC59, pOC60, and pOC61, using a coupled transcription and translation rabbit reticulocyte lysate system containing SP6 RNA polymerase (Promega). For degradation assays, $4 \mu$ l translation mixture were incubated at room temperature with $16 \mu \mathrm{l}$ interphase or mitotic Xenopus egg extracts (King et al. 1995), containing $100 \mu \mathrm{g} / \mathrm{ml}$ cycloheximide, $1.25 \mathrm{mg} / \mathrm{ml}$ bovine ubiquitin (Sigma), energy mix (7.5 mM creatine phosphate, $1 \mathrm{~mm} \mathrm{ATP,} 1$ $\mathrm{mm} \mathrm{MgCl}_{2}, 0.1 \mathrm{~mm}$ EGTA), and, in the case of the mitotic extract, a bacterially expressed non-degradable $\delta 90$ fragment of sea urchin cyclin B (Glotzer et al. 1991). Samples were removed at various time points, mixed with SDS sample buffer and analyzed by SDS-polyacrylamide gel electrophoresis (PAGE) and an ImageQuant analysis using a Molecular Dynamics PhosphorImager. Immunodepletions of extracts with $\mathrm{Cdc} 27 \mathrm{p}$ antibodies (Tugendreich et al. 1995) or preimmune antibodies were done as described (King et al. 1995). For ubiquitination assays, $2 \mu \mathrm{l}$ of the translation mixture were incubated for $20 \mathrm{~min}$ at room temperature in a $10-\mu \mathrm{l}$ reaction mixture containing $5 \mu \mathrm{l}$ of immunopurified mitotic Xenopus APC bound to CDC27 antibody beads (King et al. 1995), $1.25 \mathrm{mg} / \mathrm{ml}$ bovine ubiquitin bacterially expressed and purified, wheat E1 and either Xenopus UBC4 or UBCx (50 $\mu \mathrm{g} / \mathrm{ml} \mathrm{each)} \mathrm{(Yu} \mathrm{et} \mathrm{al.} \mathrm{1996),} \mathrm{and} 10 \mathrm{~mm}$ Tris-HCl (pH 7.7), $100 \mathrm{mM} \mathrm{KCl}, 1 \mathrm{mM} \mathrm{MgCl}_{2}$ and energy mix. Reactions were stopped by addition of SDS sample buffer and analyzed as above. Methylated ubiquitin was the kind gift of Renee Yew (Harvard Medical School, Cambridge, MA) and was used at a concentration of $3 \mathrm{mg} / \mathrm{ml}$. An in vitro translated fusion protein consisting of the amino-terminal residues 13-92 of sea urchin cyclin B fused to protein A (13-91-prA; Glotzer et al. 1991) was used as a positive control in degradation and ubiquitination assays. Quantitation of the distribution of the radiolabeled bands was done by using the ImageQuant program version 1.1 from Molecular Dynamics (Sunnyvale, CA).

Other methods

Isolation of RNA and Northern blot analysis were carried out as described (Megee et al. 1995). PDS1 mRNA was detected using a radiolabeled DNA fragment containing the PDS1 open reading frame. $A C T 1$ mRNA was detected using a radiolabeled EcoRI$B a m H I$ fragment containing an intronless $A C T 1$ gene from plasmid KCe44 (Chapman and Boeke 1991) (a generous gift from J. 
Boeke, Johns Hopkins University Medical School, Baltimore, MD). Labeling was done using the Random Primer DNA labeling Kit (Boehringer Mannheim).

For Western blot analysis, protein extracts were prepared as described (Payne et al. 1987) and electro blotting was done onto a Protran membrane (Schleicher and Schuell, Keene, NH). The primary antibodies used were rabbit polyclonal anti-Pdslp (Yamamoto et al. 1996a), 12CA5 monoclonal antibody against the hemagglutinin epitope (BAbCO, Richmond, $\mathrm{CA}$ ); and rabbit polyclonal 206-1 raised against the last 12 carboxy-terminal amino acids of yeast $\beta$-tubulin (a generous gift from Frank Solomon, MIT, Cambridge, MA). The secondary antibodies used were horseradish peroxydase-conjugated goat antirabbit or goat antimouse antibodies (Bio-Rad, Hercules, CA). The protein bands were detected by the Renaissance Chemiluminescence reagent (DuPont NEN, Boston, MA). To quantify the chemiluminescent signal, the autoradiographs were scanned and analyzed using ImageQuant. Several different exposures of each gel were analyzed to confirm that the measurements were within the linear signal range. Indirect immunofluorescence was carried out as described (Kilmartin and Adams 1984). When Pds lpHA was visualized, cells were fixed for 20-25 min only flonger fixation times resulted in loss of HA-dependent immunofluorescence). The primary antibodies used were 12CA5 monoclonal antibody against hemagglutinin antigen (BAbCO, Richmond CA), and YOL1/34 monoclonal antibody against $\alpha$ tubulin (Serotec, Indianapolis, IN). Fluorescent in situ hybridization (FISH) was carried out as described (Guacci et al. 1994).

\section{Acknowledgments}

We wish to thank Kim Nasmyth, Vincent Guacci, Jef Boeke, and Reed Wickner for strains and plasmids, and Frank Solomon for the anti $\beta$-tubulin antibodies. We thank Jim Flook for operating the flow cytometer and Eileen Hogan and Paul Megee for assistance with the FISH experiment. We wish to thank Mitsuhiro Yanagida and David Pellman for providing data prior to publication. J.M.P and M.W.K thank Hongtao Yu for UBCx, Renee Yew for methylated ubiquitin, and Phil Hieter for CDC27 antibodies. We thank Andy Hoyt, Kim Nasmyth, David Pellman, and the members of the Koshland lab for helpful discussions and critical reading of this manuscript. This work was supported by a Human Frontier Science Program Fellowship LT-532/94 to O.C.F., grant GM41718-06 from the National Institutes of Health (NIH) to D.K., and grants GM39023-08 and GM26875-17 from the NIH to M.W.K.

The publication costs of this article were defrayed in part by payment of page charges. This article must therefore be hereby marked "advertisement" in accordance with 18 USC section 1734 solely to indicate this fact.

\section{References}

Amon, A., S. Irniger, and K. Nasmyth. 1994. Closing the cell cycle circle in yeast: $\mathrm{G} 2$ cyclin proteolysis initiated at mitosis persists until the activation of Gl cyclins in the next cycle. Cell 77: 1037-1050.

Chapman, K.B. and J.D. Boeke. 1991. Isolation and characterization of the gene encoding yeast debranching enzyme. Cell 65: $483-492$.

Chau, V., J.W. Tobias, A. Bachmair, D. Marriott, D.J. Eckler, D.K. Gonda, and A. Varshavsky. 1989. A multiubiquitin chain is confined to specific lysine in a targeted short-lived protein. Science 243: 1576-1583.

Ciechanover, A. 1994. The ubiquitin-proteasome proteolytic pathway. Cell 79: 13-21.

Culotti, J. and L.H. Hartwell. 1971. Genetic control of the cell division cycle in yeast. Exp. Cell Res. 67: 389-401.

Funabiki, H., K. Kumada, and M. Yanagida. 1996a. Fission yeast Cut 1 and Cut 2 are essential for sister chromatid separation, concentrate along the metaphase spindle and form large complexes. $E M B O J$. (in press).

Funabiki, H., H. Yamano, K. Kumada, K. Nagao, T. Hunt, and M. Yanagida. 1996. Cut2 proteolysis required for sister chromatid separation in fission yeast. Nature 381: 438-441.

Glotzer, M., A.W. Murray, and M.W. Kirschner. 1991. Cyclin is degraded by the ubiquitin pathway. Nature 349: 132-138.

Guacci, V., E. Hogan, and D. Koshland. 1994. Chromosome condensation and sister chromatid pairing in budding yeast. $/$. Cell Biol. 125: 517-513.

Hartwell, L.H. and M.B. Kastan. 1994. Cell cycle control and cancer. Science 266: 1821-1828.

Hershko, A., D. Ganoth, V. Sudakin, A. Dahan, L.H. Cohen, F.C. Luca, J.V. Ruderman, and E. Eytan. 1994. Components of a system that ligates cyclin to ubiquitin and their regulation by the protein kinase Cdc2. J. Biol. Chem. 269: 49404946.

Hirano, T., Y. Hiraoka, and M. Yanagida. 1988. A temperaturesensitive mutation of the Schizosaccharomyces pombe gene nuc2 + that encodes a nuclear-scaffold protein blocks spindle elongation in mitotic anaphase. $J$. Cell Biol. 106: 11711183.

Hirano, T., N. Kinoshita, K. Morikawa, and M. Yanagida. 1990. Snap helix with knob and hole: Essential repeats in S. pombe nuclear protein $\mathrm{nuC2}^{+}$. Cell 60: 319-328.

Holloway, S.L., M. Glotzer, R.W. King, and A.W. Murray. 1993. Anaphase is initiated by proteolysis rather than by the inactivation of maturation-promoting factor. Cell 73: 13931402.

Holm, C. 1994. Coming undone: How to untangle a chromosome. Cell 77: 955-957.

Icho, T. and R.B. Wickner. 1987. Metal-binding, nucleic acidbinding finger sequences in the CDC16 gene of Saccharomyces cerevisiae. Nucleic Acids Res. 15: 8439-8450.

Irniger, S., S. Piatti, C. Michaelis, and K. Nasmyth. 1995. Genes involved in sister chromatid separation are needed for B-type cyclin proteolysis in budding yeast. Cell 61: 269-277.

Kilmartin, J. and A. Adams. 1984. Structural rearrangements of tubulin and actin during the cell cycle of the yeast Saccharomyces. I. Cell Biol. 98: 922-933.

King, R.W., J.-M. Peter, S. Tugendreich, M. Rolfe, P. Hieter, and M.W. Kirschner. 1995. A 20 S complex containing CDC27 and $\mathrm{CDCl} 16$ catalyzes the mitosis-specific conjugation of ubiquitin to cyclin B. Cell 81: 279-288.

Lamb, J.R., W. Michaud, R.S. Sikorski, and P.A. Hieter. 1994. Cdcl6p, Cdc23p and Cdc27p form a complex essential for mitosis. $E M B O$ I. 13: 4321-4328.

Luca, F.C., E.K. Shibuya, C.E. Dohrmann, and J.V. Ruderman. 1991. Both cyclin $A \Delta 60$ and $B \Delta 97$ are stable and arrest cells in $\mathrm{M}$-phase, but only cyclin B $\Delta 97$ turns on cyclin destruction. EMBO I. 19: 4311-4320.

McGrew, J.T., L. Goetsch, B. Byers, and P. Baum. 1992. Requirement for ESP1 in the nuclear division of Saccharomyces cerevisiae. Mol. Biol. Cell 3: 1443-1454.

Megee, P.C., B.A. Morgan, and M.M. Smith. 1995. Histone H4 and the maintenance of genome integrity. Genes \& Dev. 9: $1716-1727$.

Miyazaki, W.Y. and T.L. Orr-Weaver. 1994. Sister-chromatid cohesion in mitosis and meiosis. Annu. Rev. Gen. 28: 167187.

Mumberg, D., R. Muller, and M. Funk. 1994. Regulatable pro- 
moters of Saccharomyces cerevisiae: Comparison of transcriptional activity and their use for heterologous expression. Nucleic Acid Res. 22: 5767-5768.

Murray, A. 1995. Cyclin ubiquitination: The destructive end of mitosis. Cell 81: 149-152.

Murray, A.W. and M.W. Kirschner. 1989. Cyclin synthesis drives the early embryonic cell cycle. Nature 339: 275-280.

Murray, A.W., M.J. Solomon, and M.W. Kirschner. 1989. The role of cyclin synthesis and degradation in the control of maturation promoting factor activity. Nature 339: 280-286.

O'Donnell, K.L., A.H. Osmani, S.A. Osmani, and N.R. Morris. 1991. bimA encodes a member of the tetratricopeptide repeat family of proteins and is required for the completion of mitosis in Aspergillus nidulans. I. Cell Sci. 99: 711-719.

Payne, G.S., T.B. Hasson, M.S. Hasson, and R. Schekman. 1987. Genetic and biochemical characterization of clathrin-deficient Saccharomyces cerevisiae. Mol. Cell Biol. 7: 38883898.

Peters, J.-M. 1994. Proteasomes: Protein degradation machines of the cell. Trends. Biochem. Sci. 19: 377-382.

Rose, M.D., F. Winston, and P. Hieter. 1990. Methods in yeast genetics, a laboratory course manual. Cold Spring Harbor Laboratory Press, Cold Spring Harbor, NY.

Sikorski, R.S., M. Boguski, M. Goebl, and P. Hieter. 1990. A repeating amino acid motif in CDC23 defines a family of proteins and a new relationship among genes required for mitosis and RNA synthesis. Cell 60: 307-317.

Stueland, C.S., D.J. Lew, M.J. Cismowski, and S.I. Reed. 1993. Full activation of p34CDC28 histone $\mathrm{Hl}$ kinase activity is unable to promote entry into mitosis in checkpoint-arrested cells of the yeast Saccharomyces cerevisiae. Mol. Cell Biol. 13: 3744-3755.

Sudakin, V., D. Ganoth, A. Dahan, H. Heller, I. Hershko, F.C. Luca, J.V. Ruderman, and A. Hershko. 1995. The cyclosome, a large complex containing cyclin-selective ubiquitin ligase activity, targets cyclins for destruction at the end of mitosis. Mol. Biol. Cell 6: 185-198.

Surana, U., A. Amon, C. Dowzer, I. McGrew, B. Byers, and K. Nasmyth. 1993. Destruction of the CDC28/CLB mitotic kinase is not required for the metaphase to anaphase transition in budding yeast. EMBO J. 12: 1969-1978.

Tugendreich, S., M.S. Boguski, M.S. Seldin, and P. Hieter. 1993. Linking yeast genetics to mammalian genomes: Identification and mapping of the human homolog of $\mathrm{CDC} 27$ via the expressed sequence tag (EST) data base. Proc. Natl. Acad. Sci. 90: 10031-10035.

Tugendreich, S., J. Tomkiel, W. Earnshaw, and P. Hieter. 1995. $\mathrm{CDC} 27 \mathrm{Hs}$ colocalizes with $\mathrm{CDC} 16 \mathrm{Hs}$ to the centrosome and mitotic spindle and is essential for the metaphase to anaphase transition. Cell 81: 261-268.

Turner, D.L. and H. Weintraub. 1994. Expression of achaetescute homolog 3 in Xenopus embryos converts ectodermal cells to a neural fate. Genes \& Dev. 8: 1434-1447.

Uzawa, S., I. Samejima, T. Hirano, K. Tanaka, and M. Yanagida. 1990. The fission yeast $\mathrm{cut}^{+}$gene regulates spindle pole body duplication and has homology to the budding yeast ESP1 gene. Cell 62: 913-925.

van der Velden, H.M.W. and M.J. Lohka. 1993. Mitotic arrest caused by the amino terminus of Xenopus cyclin B2. Mol. Cell Biol. 13: 1480-1488.

Yamamoto, A., V. Guacci, and D. Koshland. 1996a. Pdslp is required for faithful execution of anaphase in yeast, Saccharomyces cerevisiae. J. Cell Biol. 133: 85-97.

. 1996b. Pdslp, an inhibitor of anaphase in budding yeast, plays a critical role in the APC and checkpoint pathway(s). I. Cell Biol. 133: 99-110.
Yu, H., R.W. King, J.-M. Peters, and M.W. Kirschner. 1996. Identification of a novel ubiquitin-conjugating enzyme involved in mitotic cyclin degradation. Curr. Biol. 6: 455-466.

Zachariae, W. and K. Nasmyth. 1996. TPR proteins required for anaphase progression mediate ubiquitination of mitotic B-type cyclins in yeast. Mol. Biol. Cell 7: 791-801. 


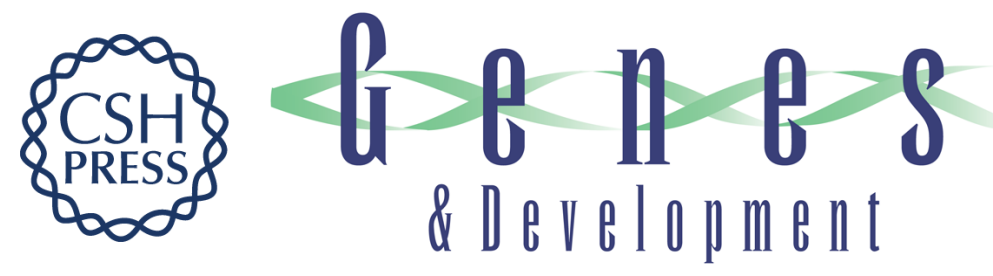

\section{Anaphase initiation in Saccharomyces cerevisiae is controlled by the APC-dependent degradation of the anaphase inhibitor Pds1p.}

O Cohen-Fix, J M Peters, M W Kirschner, et al.

Genes Dev. 1996, 10:

Access the most recent version at doi:10.1101/gad.10.24.3081

References This article cites 42 articles, 17 of which can be accessed free at:

http://genesdev.cshlp.org/content/10/24/3081.full.html\#ref-list-1

License

Email Alerting

Service

Receive free email alerts when new articles cite this article - sign up in the box at the top right corner of the article or click here.

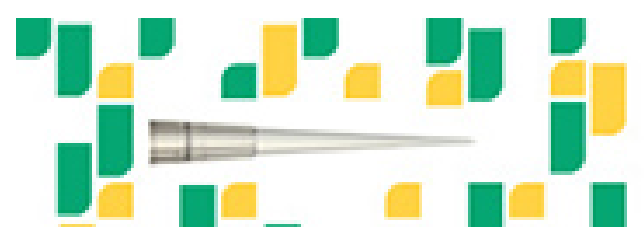

Focused on your science. 